\title{
Infinitely Many Solutions of Superlinear Elliptic Equation
}

\author{
Anmin Mao and Yang Li \\ School of Mathematical Sciences, Qufu Normal University, Jining, Shandong 273165, China \\ Correspondence should be addressed to Anmin Mao; maoam@163.com \\ Received 4 January 2013; Revised 27 April 2013; Accepted 28 April 2013 \\ Academic Editor: Wenming Zou \\ Copyright (C) 2013 A. Mao and Y. Li. This is an open access article distributed under the Creative Commons Attribution License, \\ which permits unrestricted use, distribution, and reproduction in any medium, provided the original work is properly cited. \\ Via the Fountain theorem, we obtain the existence of infinitely many solutions of the following superlinear elliptic boundary value \\ problem: $-\Delta u=f(x, u)$ in $\Omega, u=0$ on $\partial \Omega$, where $\Omega \subset \mathbb{R}^{N}(N>2)$ is a bounded domain with smooth boundary and $f$ is odd in \\ $u$ and continuous. There is no assumption near zero on the behavior of the nonlinearity $f$, and $f$ does not satisfy the Ambrosetti- \\ Rabinowitz type technical condition near infinity.
}

\section{Introduction}

Consider the following nonlinear problem:

$$
\begin{gathered}
-\Delta u=f(x, u) \quad \text { in } \Omega, \\
u=0 \quad \text { on } \partial \Omega,
\end{gathered}
$$

which has been receiving much attention during the last several decades. Here $\Omega \subset \mathbb{R}^{N}(N>2)$ is a bounded smooth domain and $f$ is a continuous function on $\Omega \times R$ and odd in $u$. We make the following assumptions on $f$ :

$\left(S_{1}\right)$ there exist constants $a_{1}>0$ and $2 N /(N-2)=2^{*}>$ $v>2$ such that

$$
|f(x, u)| \leq a_{1}\left(1+|u|^{\nu-1}\right), \quad \forall x \in \Omega, u \in R ;
$$

$\left(S_{2}\right) F(x, u) \geq 0$, for all $(x, u) \in \Omega \times R$, and

$$
\lim _{|u| \rightarrow \infty} \frac{F(x, u)}{u^{2}}=\infty, \quad \text { uniformly for } x \in \Omega,
$$

where $F(x, u)=\int_{0}^{u} f(x, u) d x ;$

$\left(S_{3}\right)$ there exists a constant $b>0$ such that

$\limsup _{|u| \rightarrow \infty} \frac{f(x, u) u-2 F(x, u)}{u^{2}+1}<b, \quad$ uniformly for $x \in \Omega$.
Note that Costa and Magalhães in [1] introduced a condition similar to $\left(S_{3}\right)$, which also appeared in [2].

In this paper, we will study the existence of infinitely many nontrivial solutions of (1) via a variant of Fountain theorems established by Zou in [3]. Fountain theorems and their dual form were established by Bartsch in [4] and by Bartsch and Willem in [5], respectively. They are effective tools for studying the existence of infinitely many large or small energy solutions. It should be noted that the P.S. condition and its variants play an important role for these theorems and their applications.

We state our main result as follows.

Theorem 1. Assume that $\left(S_{1}\right)-\left(S_{3}\right)$ hold and $f(x, u)$ is odd in $u$. Then problem (1) possesses infinitely many solutions.

Problem (1) was studied widely under various conditions on $f(x, u)$; see, for example, [6-10]. In 2007, Rabinowitz et al. [6] studied the problem

$$
-\Delta u=\lambda u+f(x, u) \quad \text { in } \Omega, u=0 \text { on } \partial \Omega,
$$

where $\Omega \subset R^{N}$ is a bounded smooth domain, and assumed

$$
\begin{aligned}
& \left(f_{1}\right) f \in C^{1}(\Omega \times R, R), \\
& \left(f_{2}\right) f(x, 0)=0=f_{u}(x, 0), \\
& \left(f_{3}\right)|f(x, u)| \leq C\left(1+|u|^{p-1}\right), 2<p<2^{*}, \\
& \left(f_{4}\right) \exists \mu>2, M>0 \text {, s.t. } \\
& \quad x \in \Omega, \quad|u| \geq M \Longrightarrow 0<\mu F(x, u) \leq u f(x, u),
\end{aligned}
$$


$\left(f_{5}\right) F(x, u) \geq 0$, for all $x$ and $u$, and $u f(x, u)>0$ for $|u|>$ 0 small.

They got the existence of at least three nontrivial solutions. $\left(f_{4}\right)$ was given by Ambrosetti and Rabinowitz [11] to ensure that some compactness and the Mountain Pass setting hold.

However, there are many functions which are superlinear but do not necessarily need to satisfy $\left(f_{4}\right)$. For example,

$$
F(x, u)=\frac{1}{2} u^{2} \ln (1+u)-\frac{1}{2}\left(\frac{u^{2}}{2}-u+\ln (1+u)\right) .
$$

It is easy to check that $\left(f_{4}\right)$ does not hold. On the other hand, in order to verify $\left(f_{4}\right)$, it is usually an annoying task to compute the primitive function of $f$ and sometimes it is almost impossible, for example,

$$
f(x, u)=|u| u\left(1+e^{(1+|\sin u|)^{\alpha}}+|\cos u|^{\alpha}\right), \quad u \in R, \alpha>0 .
$$

More examples are presented in Remark 2.

We recall that $\left(f_{4}\right)$ implies a weaker condition

$$
F(x, u) \geq c|u|^{\theta}-d, \quad c, d>0 \text {, a.e. } x \in \Omega, u \in R, \theta>2 \text {. }
$$

In [12], Willem and Zou gave one weaker condition

$$
c|u|^{\theta} \leq u f(x, u) \quad \text { for }|u| \geq R_{0} \text {, a.e. } x \in \Omega, \theta>2 .
$$

Note that $\left(S_{2}\right)$ is much weaker than the above conditions.

In [13], Schechter and Zou proved that under the hypotheses that

$\left(S_{1}\right)$ holds and

$$
\text { either } \lim _{u \rightarrow-\infty} \frac{F(x, u)}{u^{2}}=+\infty \text { or } \lim _{u \rightarrow \infty} \frac{F(x, u)}{u^{2}}=+\infty \text {, }
$$

problem (1) has a nontrivial weak solution.

Recently, Miyagaki and Souto in [2] proved that problem (1) has a nontrivial solution via the Mountain Pass theorem under the following conditions:

$\left(S_{1}\right)$ and $\left(S_{2}\right)$ hold, and

$$
\begin{aligned}
& \lim _{|u| \rightarrow 0} \frac{f(x, u)}{u}=0, \quad \exists u_{0}>0, \\
& \text { s.t. } \frac{f(x, u)}{u} \text { is increasing in } u \geq u_{0} \\
& \text { and decreasing in } u \leq-u_{0}, \forall x \in \Omega,
\end{aligned}
$$

and they adapted some monotonicity arguments used by Schechter and Zou [13]. This approach is interesting, but many powerful variational tools such as the Fountain theorem and Morse theory are not directly applicable. In addition, the monotonicity assumption on $F(x, u) / u^{2}$ is weaker than the monotonicity assumption on $f(x, u) / u$.

As to the case in the current paper, we make some concluding remarks as follows.
Remark 2. To show that our assumptions $\left(S_{2}\right)$ and $\left(S_{3}\right)$ are weaker than $\left(f_{4}\right)$, we give two examples:

(1) $f(x, u)=2 u \ln u+u$,

(2) $f(x, u)=\gamma|u|^{\gamma-2} u+(\gamma-1)|u|^{\gamma-3} u \sin ^{2} u+|u|^{\gamma-1} \sin 2 u$, $u \in R \backslash\{0\}, \gamma>2$,

which do not satisfy $\left(f_{4}\right)$. Example (2) can be found in [3]. So the case considered here cannot be covered by the cases mentioned in $[6,11]$.

Remark 3. Compared with papers [11, 12], we do not assume any superlinear conditions near zero. Compared with paper [2], we do not impose any kind of monotonic conditions. In addition, although we do not assume $\left(f_{4}\right)$ holds, we are able to check the boundedness of P.S. (or P.S. ${ }^{*}$ ) sequences. So, our result is different from those in the literature.

Our argument is variational and close to that in $[2,3$, 13, 14]. The paper is arranged as follows. In Section 2 we formulate the variational setting and recall some critical point theorems required. We then in Section 3 complete the proof of Theorem 1 .

\section{Variational Setting}

In this section, we will first recall some related preliminaries and establish the variational setting for our problem. Throughout this paper, we work on the space $E=H_{0}^{1}(\Omega)$ equipped with the norm

$$
\|u\|=\left(\int_{\Omega}|\nabla u|^{2} d x\right)^{1 / 2} .
$$

Lemma 4. E embeds continuously into $L^{p}$, for all $0<p \leq 2^{*}$, and compactly into $L^{p}$, for all $1 \leq p<2^{*}$; hence there exists $\tau_{p}>0$ such that

$$
|u|_{p} \leq \tau_{p}\|u\|, \quad \forall u \in E
$$

where $|u|_{p}=\left(\int_{\Omega}|u|^{p} d x\right)^{1 / p}$.

Define the Euler-Lagrange functional associated to problem (1), given by

$$
I(u)=\frac{1}{2}\|u\|^{2}-\Psi(u), \quad u \in E,
$$

where $\Psi(u)=\int_{\Omega} F(x, u) d x$. Note that $\left(S_{1}\right)$ implies that

$$
F(x, u) \leq a_{1}\left(|u|+|u|^{\nu}\right), \quad \forall(x, u) \in \Omega \times R .
$$

In view of (16) and Sobolev embedding theorem, $I_{\mu}$ and $\Psi$ are well defined. Furthermore, we have the following.

Lemma 5 (see [15] or [16]). Suppose that $\left(S_{1}\right)$ is satisfied. Then $\Psi \in C^{1}(E, R)$ and $\Psi^{\prime}: E \rightarrow E^{*}$ is compact and hence $I \in$ $C^{1}(E, R)$. Moreover

$$
\begin{gathered}
\Psi^{\prime}(u) v=\int_{\Omega} f(x, u) v d x, \\
I^{\prime}(u) v=\int_{\Omega} \nabla u \nabla v d x-\Psi^{\prime}(u) v,
\end{gathered}
$$

for all $u, v \in E$, and critical points of I on E are solutions of (1). 
Lemma 6 (see [17]). Assume that $|\Omega|<\infty, 1 \leq p, r \leq$ $\infty, f \in C(\bar{\Omega} \times R)$, and $|f(x, u)| \leq c\left(1+|u|^{p / r}\right)$. Then for every $u \in L^{p}(\Omega), f(x, u) \in L^{r}(\Omega)$, and the operator $A: L^{p}(\Omega) \mapsto$ $L^{r}(\Omega): u \mapsto f(x, u)$ is continuous.

Let $E$ be a Banach space equipped with the norm $\|\cdot\|$ and $E=\overline{\bigoplus_{j \in N} X_{j}}$, where $\operatorname{dim} X_{j}<\infty$ for any $j \in N$. Set $Y_{k}=\bigoplus_{j=1}^{k} X_{j}$ and $Z_{k}=\overline{\bigoplus_{j=k}^{\infty} X_{j}}$. Consider the following $C^{1}$ functional $\Phi_{\lambda}: E \rightarrow R$ defined by

$$
\Phi_{\lambda}(u):=A(u)-\lambda B(u), \quad \lambda \in[1,2] .
$$

The following variant of the Fountain theorems was established in [3].

Theorem 7 (see [3, Theorem 2.1]). Assume that the functional $\Phi_{\lambda}$ defined above satisfies the following:

$\left(F_{1}\right) \Phi_{\lambda}$ maps bounded sets to bounded sets uniformly for $\lambda \in[1,2]$; furthermore, $\Phi_{\lambda}(-u)=\Phi_{\lambda}(u)$ for all $(\lambda, u) \in[1,2] \times E$

$\left(F_{2}\right) B(u) \geq 0$ for all $u \in E$; moreover, $A(u) \rightarrow \infty$ or $B(u) \rightarrow \infty$ as $\|u\| \rightarrow \infty$;

$\left(F_{3}\right)$ there exists $r_{k}>\rho_{k}>0$ such that

$$
\begin{aligned}
\alpha_{k}(\lambda) & :=\inf _{u \in Z_{k},\|u\|=\rho_{k}} \Phi_{\lambda}(u)>\beta_{k}(\lambda) \\
& :=\max _{u \in Y_{k},\|u\|=r_{k}} \Phi_{\lambda}(u), \quad \forall \lambda \in[1,2] .
\end{aligned}
$$

Then

$$
\alpha_{k}(\lambda) \leq \zeta_{k}(\lambda):=\inf _{\gamma \in \Gamma_{k}} \max _{u \in B_{k}} \Phi_{\lambda}(\gamma(u)), \forall \lambda \in[1,2],
$$

where $B_{k}=\left\{u \in Y_{k}:\|u\| \leq r_{k}\right\}$ and $\Gamma_{k}:=\{\gamma \in$ $C\left(B_{k}, E\right) \mid \gamma$ is odd, $\left.\left.\gamma\right|_{\partial B_{k}}=i d\right\}$. Moreover, for a.e. $\lambda \in[1,2]$, there exists a sequence $\left\{u_{m}^{k}(\lambda)\right\}_{m=1}^{\infty}$ such that

$$
\begin{gathered}
\sup _{m}\left\|u_{m}^{k}(\lambda)\right\|<\infty, \quad \Phi_{\lambda}^{\prime}\left(u_{m}^{k}(\lambda)\right) \longrightarrow 0, \\
\Phi_{\lambda}\left(u_{m}^{k}(\lambda)\right) \longrightarrow \zeta_{k}(\lambda) \quad \text { as } m \longrightarrow \infty .
\end{gathered}
$$

In order to apply the above theorem to prove our main results, we define the functionals $A, B$, and $I_{\lambda}$ on our working space $E$ by

$$
\begin{gathered}
A(u)=\frac{1}{2}\|u\|^{2}, \quad B(u)=\int_{\Omega} F(x, u) d x, \\
I_{\lambda}(u)=A(u)-\lambda B(u)=\frac{1}{2}\|u\|^{2}-\lambda \int_{\Omega} F(x, u) d x,
\end{gathered}
$$

for all $u \in E$ and $\lambda \in[1,2]$. Note that $I_{1}=I$, where $I$ is the functional defined in (15).

From Lemma 5, we know that $I_{\lambda} \in C^{1}(E, R)$, for all $\lambda \in[1,2]$. It is known that $-\Delta$ is a selfadjoint operator with a sequence of eigenvalues (counted with multiplicity)

$$
0<\lambda_{1}<\lambda_{2} \leq \lambda_{3} \leq \cdots \leq \lambda_{j} \leq \cdots \longrightarrow \infty,
$$

and the corresponding system of eigenfunctions $\left\{e_{j}: j \in\right.$ $N\}\left(-\Delta e_{j}=\lambda_{j} e_{j}\right)$ forming an orthogonal basis in $E$. Let $X_{j}=\operatorname{span}\left\{e_{j}\right\}$, for all $j \in N$.

\section{Proof of Theorem 1}

Lemma 8. Assume that $\left(S_{1}\right)-\left(S_{2}\right)$ hold. Then there exists a positive integer $k_{1}$ and two sequences $r_{k}>\rho_{k} \rightarrow \infty$ as $k \rightarrow \infty$ such that

$$
\begin{aligned}
& \alpha_{k}(\lambda):=\inf _{u \in Z_{k},\|u\|=\rho_{k}} I_{\lambda}(u)>0, \quad \forall k \geq k_{1}, \\
& \beta_{k}(\lambda):=\max _{u \in Y_{k},\|u\|=r_{k}} I_{\lambda}(u)<0, \quad \forall k \in N,
\end{aligned}
$$

where $Y_{k}=\bigoplus_{j=1}^{k} X_{j}=\operatorname{span}\left\{e_{1}, \ldots, e_{k}\right\}$ and $Z_{k}=\overline{\bigoplus_{j=k}^{\infty} X_{j}}=$ $\overline{\operatorname{span}\left\{e_{k}, \ldots\right\}}$, for all $k \in N$.

Proof

Step 1. We first prove (25).

By (16) and (23), for all $\lambda \in[1,2]$ and $u \in E$, we have

$$
\begin{aligned}
I_{\lambda}(u) & \geq \frac{1}{2}\|u\|^{2}-2 \int_{\Omega} a_{1}\left(|u|+|u|^{\nu}\right) d x \\
& =\frac{1}{2}\|u\|^{2}-2 a_{1}\left(|u|_{1}+|u|_{\nu}^{\nu}\right),
\end{aligned}
$$

where $a_{1}$ is the constant in (16). Let

$$
\sigma_{\nu}(k)=\sup _{u \in Z_{k},\|u\|=1}|u|_{v}, \quad \forall k \in N .
$$

Then

$$
\sigma_{\nu}(k) \longrightarrow 0 \quad \text { as } k \longrightarrow \infty,
$$

since $E$ is compactly embedded into $L^{\nu}$. Combining (14), (27), and (28), we have

$$
\begin{array}{r}
I_{\lambda} \geq \frac{1}{2}\|u\|^{2}-2 a_{1} \tau_{1}\|u\|-2 a_{1} \sigma_{v}^{\nu}(k)\|u\|^{\nu}, \\
\forall(\lambda, u) \in[1,2] \times Z_{k} .
\end{array}
$$

By (29), there exists a positive integer $k_{1}>0$ such that

$$
\rho_{k}:=\left(16 a_{1} \sigma_{\nu}^{\nu}(k)\right)^{1 /(2-\nu)}>16 a_{1} \tau_{1}, \quad \forall k \geq k_{1},
$$

since $v>2$. Evidently,

$$
\rho_{k} \longrightarrow \infty \quad \text { as } k \longrightarrow \infty \text {. }
$$

Combining (30) and (31), direct computation shows

$$
\alpha_{k}:=\inf _{u \in Z_{k},\|u\|=\rho_{k}} I_{\lambda}(u) \geq \frac{\rho_{k}^{2}}{4}>0, \quad \forall k \geq k_{1} .
$$

Step 2. We then verify (26).

We claim that for any finite-dimensional subspace $F \subset E$, there exists a constant $\epsilon>0$ such that

$$
m(\{x \in \Omega:|u(x)| \geq \epsilon\|u\|\}) \geq \epsilon, \quad \forall u \in F \backslash\{0\} .
$$

Here and in the sequel, $m(\cdot)$ always denotes the Lebesgue measure in $R$. 
If not, for any $n \in N$, there exists $u_{n} \in F \backslash\{0\}$ such that

$$
m\left(\left\{x \in \Omega:\left|u_{n}(x)\right| \geq \frac{1}{n}\left\|u_{n}\right\|\right\}\right)<\frac{1}{n} .
$$

Let $v_{n}=u_{n} /\left\|u_{n}\right\| \in F$, for all $n \in N$. Then $\left\|v_{n}\right\|=1$, for all $n \in N$, and

$$
m\left(\left\{x \in \Omega:\left|v_{n}(x)\right| \geq \frac{1}{n}\right\}\right)<\frac{1}{n}, \quad \forall n \in N .
$$

Passing to a subsequence if necessary, we may assume $v_{n} \rightarrow$ $v_{0}$ in $E$, for some $v_{0} \in F$, since $F$ is of finite dimension. Evidently, $\left\|v_{0}\right\|=1$. In view of Lemma 4 and the equivalence of any two norms on $F$, we have

$$
\int_{\Omega}\left|v_{n}-v_{0}\right| d x \rightarrow 0 \quad \text { as } n \longrightarrow \infty
$$

and $\left|v_{0}\right|_{\infty}>0$.

By the definition of norm $|\cdot|_{\infty}$, there exists a constant $\delta_{0}>0$ such that

$$
m\left(\left\{x \in \Omega:\left|v_{0}(x)\right| \geq \delta_{0}\right\}\right) \geq \delta_{0} .
$$

For any $n \in N$, let

$$
\begin{gathered}
\Lambda_{n}=\left\{x \in \Omega:\left|v_{n}(x)\right|<\frac{1}{n}\right\}, \\
\Lambda_{n}^{c}=\Omega \backslash \Lambda_{n}=\left\{x \in \Omega:\left|v_{n}(x)\right| \geq \frac{1}{n}\right\} .
\end{gathered}
$$

Set $\Lambda_{0}=\left\{x \in \Omega:\left|v_{0}(x)\right| \geq \delta_{0}\right\}$. Then for $n$ large enough, by (36) and (38), we have

$$
\begin{aligned}
m\left(\Lambda_{n} \cap \Lambda_{0}\right) & =m\left(\Lambda_{0} \backslash \Lambda_{n}^{c}\right) \\
& \geq m\left(\Lambda_{0}\right)-m\left(\Lambda_{n}^{c}\right) \geq \delta_{0}-\frac{1}{n} \geq \frac{\delta_{0}}{2} .
\end{aligned}
$$

Consequently, for $n$ large enough, there holds

$$
\begin{aligned}
\int_{\Omega}\left|v_{n}-v_{0}\right| d x & \geq \int_{\Lambda_{n} \cap \Lambda_{0}}\left|v_{n}-v_{0}\right| d x \\
& \geq \int_{\Lambda_{n} \cap \Lambda_{0}}\left(\left|v_{0}\right|-\left|v_{n}\right|\right) d x \\
& \geq\left(\delta_{0}-\frac{1}{n}\right) m\left(\Lambda_{n} \cap \Lambda_{0}\right) \\
& \geq \frac{\delta_{0}^{2}}{4}>0 .
\end{aligned}
$$

This is in contradiction to (37). Therefore (34) holds.

Consequently, for any $k \in N$, there exists a constant $\epsilon_{k}>$ 0 such that

$$
m\left(\Lambda_{u}^{k}\right) \geq \epsilon_{k}, \quad \forall u \in Y_{k} \backslash\{0\},
$$

where $\Lambda_{u}^{k}:=\left\{x \in \Omega:|u(x)| \geq \epsilon_{k}\|u\|\right\}$, for all $k \in N$, and $u \in Y_{k} \backslash\{0\}$. By $\left(S_{2}\right)$, for any $k \in N$, there exists a constant $S_{k}>0$ such that

$$
F(x, u) \geq \frac{|u|^{2}}{\epsilon_{k}^{3}}, \quad \forall|u| \geq S_{k}
$$

Combining (23), (42), (43), and $\left(S_{2}\right)$, for any $k \in N$ and $\lambda \epsilon$ $[1,2]$, we have

$$
\begin{aligned}
I_{\lambda}(u) & \leq \frac{1}{2}\|u\|^{2}-\int_{\Lambda_{u}^{k}} \frac{|u|^{2}}{\epsilon_{k}^{3}} d x \\
& \leq \frac{1}{2}\|u\|^{2}-\frac{1}{\epsilon_{k}^{3}} \epsilon_{k}^{2}\|u\|^{2} m\left(\Lambda_{u}^{k}\right) \\
& \leq \frac{1}{2}\|u\|^{2}-\|u\|^{2} \\
& =-\frac{1}{2}\|u\|^{2}
\end{aligned}
$$

with $\|u\| \geq S_{k} / \epsilon_{k}$. Now for any $k \in N$, if we choose

$$
r_{k}>\max \left\{\rho_{k}, \frac{S_{k}}{\epsilon_{k}}\right\},
$$

then (44) implies

$$
\beta_{k}(\lambda):=\max _{u \in Y_{k},\|u\|=r_{k}} I_{\lambda}(u) \leq-\frac{r_{k}^{2}}{2}<0, \quad \forall k \in N,
$$

ending the proof.

Proof of Theorem 1. It follows from (16), (23), and Lemma 5 that $I_{\lambda}$ maps bounded sets to bounded sets uniformly for $\lambda \in[1,2]$. In view of the evenness of $F(x, u)$ in $u$, it holds that $I_{\lambda}(-u)=I_{\lambda}(u)$ for all $(\lambda, u) \in[1,2] \times E$. Thus the condition $\left(F_{1}\right)$ of Theorem 7 holds. Besides, $A(u)=(1 / 2)\|u\|^{2} \rightarrow \infty$ as $\|u\| \rightarrow \infty$ and $B(u) \geq 0$ since $F(x, u) \geq 0$. Thus the condition $\left(F_{2}\right)$ of Theorem 7 holds. And Lemma 8 shows that the condition $\left(F_{3}\right)$ holds for all $k \geq k_{1}$. Therefore, by Theorem 7, for any $k \geq k_{1}$ and a.e. $\lambda \in[1,2]$, there exists a sequence $\left\{u_{m}^{k}(\lambda)\right\}_{m=1}^{\infty} \subset E$ such that

$$
\begin{gathered}
\sup _{m}\left\|u_{m}^{k}(\lambda)\right\|<\infty, \quad I_{\lambda}^{\prime}\left(u_{m}^{k}(\lambda)\right) \longrightarrow 0, \\
I_{\lambda}\left(u_{m}^{k}(\lambda)\right) \longrightarrow \zeta_{k}(\lambda)
\end{gathered}
$$

as $m \rightarrow \infty$, where

$$
\zeta_{k}(\lambda):=\inf _{h \in \Gamma_{k}} \max _{u \in B_{k}} I_{\lambda}(h(u)), \quad \forall \lambda \in[1,2],
$$

with $B_{k}=\left\{u \in Y_{k}:\|u\| \leq r_{k}\right\}$ and $\Gamma_{k}:=\left\{h \in C\left(B_{k}, E\right) \mid\right.$ $h$ is odd, $\left.\left.h\right|_{\partial B_{k}}=i d\right\}$.

Furthermore, it follows from the proof of Lemma 8 that

$$
\zeta_{k}(\lambda) \in\left[\bar{\alpha}_{k}, \bar{\zeta}_{k}\right], \quad \forall k \geq k_{1}
$$

where $\bar{\zeta}_{k}:=\max _{u \in B_{k}} I_{\lambda}(u)$ and $\bar{\alpha}_{k}:=\rho_{k}^{2} / 4 \rightarrow \infty$ as $k \rightarrow \infty$ by (32).

Claim 1. $\left\{u_{m}^{k}(\lambda)\right\}_{m=1}^{\infty} \subset E$ possesses a strong convergent subsequence in $E$, for $\forall \lambda \in[1,2]$ and $k \geq k_{1}$.

In fact, by the boundedness of the $\left\{u_{m}^{k}(\lambda)\right\}_{m=1}^{\infty}$, passing to a subsequence, as $m \rightarrow \infty$, we may assume

$$
u_{m}^{k}(\lambda) \rightarrow u^{k}(\lambda) \quad \text { in } E .
$$


By the Sobolev embedding theorem,

$$
u_{m}^{k}(\lambda) \longrightarrow u^{k}(\lambda) \quad \text { in } L^{\nu} .
$$

Lemma 6 implies that

$$
f\left(x, u_{m}^{k}(\lambda)\right) \longrightarrow f\left(x, u^{k}(\lambda)\right) \quad \text { in } L^{\nu /(\nu-1)}
$$

Observe that

$$
\begin{aligned}
& \left\|u_{m}^{k}(\lambda)-u^{k}(\lambda)\right\|^{2} \\
& =\left(I_{\lambda}^{\prime}\left(u_{m}^{k}(\lambda)\right)-I_{\lambda}^{\prime}\left(u^{k}(\lambda)\right), u_{m}^{k}(\lambda)-u^{k}(\lambda)\right) \\
& +\lambda \int_{\Omega}\left(f\left(x, u_{m}^{k}(\lambda)\right)-f\left(x, u^{k}(\lambda)\right)\right) \\
& \quad \times\left(u_{m}^{k}(\lambda)-u^{k}(\lambda)\right) d x .
\end{aligned}
$$

By (47), it is clear that

$$
\begin{aligned}
\left(I_{\lambda}^{\prime}\left(u_{m}^{k}(\lambda)\right)-I_{\lambda}^{\prime}\left(u^{k}(\lambda)\right), u_{m}^{k}(\lambda)-u^{k}(\lambda)\right) & \longrightarrow 0 \\
\text { as } m & \longrightarrow \infty .
\end{aligned}
$$

It follows from the Hölder inequality, (51), and (52) that

$$
\begin{aligned}
\mid \int_{\Omega}( & \left.f\left(x, u_{m}^{k}(\lambda)\right)-f\left(x, u^{k}(\lambda)\right)\right)\left(u_{m}^{k}(\lambda)-u^{k}(\lambda)\right) d x \mid \\
\leq & \left|f\left(x, u_{m}^{k}(\lambda)\right)-f\left(x, u^{k}(\lambda)\right)\right|_{\nu /(v-1)} \\
& \times\left|u_{m}^{k}(\lambda)-u^{k}(\lambda)\right|_{\nu} \longrightarrow 0
\end{aligned}
$$

as $m \rightarrow \infty$. Thus by (53), (54), and (55), we have proved that

$$
\left\|u_{m}^{k}(\lambda)-u^{k}(\lambda)\right\| \longrightarrow 0 \quad \text { as } m \longrightarrow \infty
$$

that is, $u_{m}^{k}(\lambda) \rightarrow u^{k}(\lambda)$ in $E$.

Thus, for each $k \geq k_{1}$, we can choose $\lambda_{n} \rightarrow 1$ such that the sequence $\left\{u_{m}^{k}\left(\lambda_{n}\right)\right\}_{m=1}^{\infty}$ obtained a convergent subsequence; passing again to a subsequence, we may assume

$$
\lim _{m \rightarrow \infty} u_{m}^{k}\left(\lambda_{n}\right)=u_{n}^{k} \quad \text { in } E, \forall n \in N, k \geq k_{1} .
$$

This together with (47) and (49) yields

$$
I_{\lambda_{n}}^{\prime}\left(u_{n}^{k}\right)=0, \quad I_{\lambda_{n}}\left(u_{n}^{k}\right) \in\left[\bar{\alpha}_{k}, \bar{\zeta}_{k}\right], \quad \forall n \in N, k \geq k_{1}
$$

Claim 2. $\left\{u_{n}^{k}\right\}_{n=1}^{\infty}$ is bounded in $E$ for all $k \geq k_{1}$.

For notational simplicity, we will set $u_{n}=u_{n}^{k}$ for all $n \in$ $N$ throughout this paragraph. If $\left\{u_{n}\right\}$ is unbounded in $E$, we define $v_{n}=u_{n} /\left\|u_{n}\right\|$. Since $\left\|v_{n}\right\|=1$, without loss of generality we suppose that there is $v \in E$ such that

$$
\begin{gathered}
v_{n} \rightarrow v \quad \text { in } E, \\
v_{n} \longrightarrow v \quad \text { in } L^{v}(\Omega), \\
v_{n}(x) \longrightarrow v(x) \quad \text { a.e. in } \Omega .
\end{gathered}
$$

Let $\Omega_{\neq}=\{x \in \Omega: v(x) \neq 0\}$. If $x \in \Omega_{\neq}$, from $\left(S_{2}\right)$ it follows that

$$
\lim _{n \rightarrow \infty} \frac{F\left(x, u_{n}(x)\right)}{u_{n}(x)^{2}} v_{n}(x)^{2}=\infty .
$$

On the other hand, after a simple calculation, we have

$$
\lim _{n \rightarrow \infty} \int_{\Omega} \frac{F\left(x, u_{n}(x)\right)}{u_{n}(x)^{2}} v_{n}(x)^{2}=\frac{1}{2} .
$$

We conclude that $\Omega_{\neq}$has zero measure and $v \equiv 0$ a.e. in $\Omega$. Moreover, from (49) and (58)

$$
\int_{\Omega} \frac{(1 / 2) f\left(x, u_{n}\right) u_{n}-F\left(x, u_{n}\right)}{u_{n}^{2}} v_{n}^{2} d x=\frac{I_{\lambda_{n}}\left(u_{n}\right)}{\lambda_{n}\left\|u_{n}\right\|^{2}}>0 .
$$

By $\left(S_{3}\right)$,

$$
\begin{gathered}
\limsup _{n \rightarrow \infty} \frac{(1 / 2) f\left(x, u_{n}\right) u_{n}-F\left(x, u_{n}\right)}{u_{n}^{2}} v_{n}^{2} \\
<\limsup _{n \rightarrow \infty} \frac{b\left(u_{n}^{2}+1\right)}{2 u_{n}^{2}} v_{n}^{2}=0
\end{gathered}
$$

which contradicts (62). Hence $\left\{u_{n}\right\}$ is bounded.

Claim 3. $\left\{u_{n}^{k}\right\}$ possesses a convergent subsequence with the limit $u^{k} \in E$ for all $k \geq k_{1}$.

In fact, by Claim 2, without loss of generality, we have assume

$$
u_{n}^{k} \rightarrow u^{k} \quad \text { as } n \longrightarrow \infty .
$$

By virtue of the Riesz Representation theorem, $I_{\lambda}^{\prime}: E \mapsto E^{*}$ and $\Psi^{\prime}: E \mapsto E^{*}$ can be viewed as $I_{\lambda}^{\prime}: E \mapsto E$ and $\Psi^{\prime}: E \mapsto$ $E$, respectively, where $E^{*}$ is the dual space of $E$. Note that

$$
0=I_{\lambda_{n}}^{\prime}\left(u_{n}^{k}\right)=u_{n}^{k}-\lambda_{n} \Psi^{\prime}\left(u_{n}^{k}\right)
$$

that is

$$
u_{n}^{k}=\lambda_{n} \Psi^{\prime}\left(u_{n}^{k}\right)
$$

By Lemma 5, $\Psi^{\prime}: E \mapsto E$ is also compact. Due to the compactness of $\Psi^{\prime}$ and (64), the right-hand side of (66) converges strongly in $E$ and hence $u_{n}^{k} \rightarrow u^{k}$ in $E$.

Now for each $k \geq k_{1}$, by (58), the limit $u^{k}$ is just a critical point of $I_{1}=I$ with $I\left(u^{k}\right) \in\left[\bar{\alpha}_{k}, \bar{\zeta}_{k}\right]$. Since $\bar{\alpha}_{k} \rightarrow \infty$ as $k \rightarrow \infty$ in (49), we get infinitely many nontrivial critical points of $I$. Therefore (1) possesses infinitely many nontrivial solutions by Lemma 5 .

\section{Acknowledgments}

The authors would like to thank the referee for valuable comments and helpful suggestions. The first author would like to acknowledge the hospitality of Professor Y. Ding of the AMSS of the Chinese Academy of Sciences, where this paper was written during his visit. Anmin Mao was supported by NSFC (11101237) and ZR2012AM006. 


\section{References}

[1] D. G. Costa and C. A. Magalhães, "Variational elliptic problems which are nonquadratic at infinity," Nonlinear Analysis. Theory, Methods \& Applications, vol. 23, no. 11, pp. 1401-1412, 1994.

[2] O. H. Miyagaki and M. A. S. Souto, "Superlinear problems without Ambrosetti and Rabinowitz growth condition," Journal of Differential Equations, vol. 245, no. 12, pp. 3628-3638, 2008.

[3] W. Zou, "Variant fountain theorems and their applications," Manuscripta Mathematica, vol. 104, no. 3, pp. 343-358, 2001.

[4] T. Bartsch, "Infinitely many solutions of a symmetric Dirichlet problem," Nonlinear Analysis. Theory, Methods \& Applications, vol. 20, no. 10, pp. 1205-1216, 1993.

[5] T. Bartsch and M. Willem, "On an elliptic equation with concave and convex nonlinearities," Proceedings of the American Mathematical Society, vol. 123, no. 11, pp. 3555-3561, 1995.

[6] P. H. Rabinowitz, J. Su, and Z.-Q. Wang, "Multiple solutions of superlinear elliptic equations," Rendiconti Lincei. Serie IX. Matematica e Applicazioni, vol. 18, no. 1, pp. 97-108, 2007.

[7] L. Jeanjean, "On the existence of bounded Palais-Smale sequences and application to a Landesman-Lazer-type problem set on $\mathbf{R}^{N}$," Proceedings of the Royal Society of Edinburgh A, vol. 129, no. 4, pp. 787-809, 1999.

[8] Y. Ding and C. Lee, "Multiple solutions of Schrödinger equations with indefinite linear part and super or asymptotically linear terms," Journal of Differential Equations, vol. 222, no. 1, pp. 137-163, 2006.

[9] H.-S. Zhou, "Positive solution for a semilinear elliptic equation which is almost linear at infinity," Zeitschrift für Angewandte Mathematik und Physik, vol. 49, no. 6, pp. 896-906, 1998.

[10] M. Schechter, "Superlinear elliptic boundary value problems," Manuscripta Mathematica, vol. 86, no. 3, pp. 253-265, 1995.

[11] A. Ambrosetti and P. H. Rabinowitz, "Dual variational methods in critical point theory and applications," Journal of Functional Analysis, vol. 14, pp. 349-381, 1973.

[12] M. Willem and W. Zou, On a Semilinear Dirichlet Problem and a Nonlinear Schrödinger Equation with Periodic Potential, vol. 305 of Séminaire de Mathématique, Université Catholique de Louvain, 2000.

[13] M. Schechter and W. Zou, "Superlinear problems," Pacific Journal of Mathematics, vol. 214, no. 1, pp. 145-160, 2004.

[14] Q. Y. Zhang and C. G. Liu, "Infinitely many periodic solutions for second order Hamiltonian systems," Journal of Differential Equations, vol. 251, no. 4-5, pp. 816-833, 2011.

[15] V. Benci and P. H. Rabinowitz, "Critical point theorems for indefinite functionals," Inventiones Mathematicae, vol. 52, no. 3 , pp. 241-273, 1979.

[16] P. H. Rabinowitz, Minimax Methods in Critical Point Theory with Applications to Differential Equations, CBMS, 1986.

[17] M. Willem, Minimax Theorems, Birkhäuser, Boston, Mass, USA, 1996. 


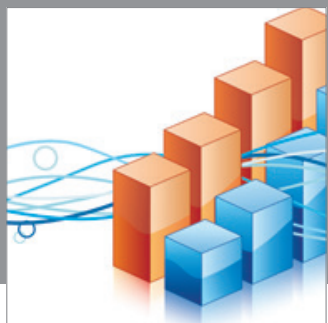

Advances in

Operations Research

mansans

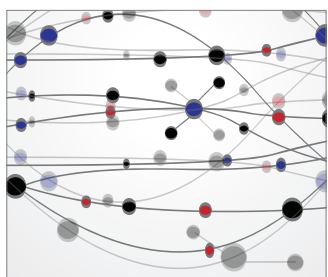

The Scientific World Journal
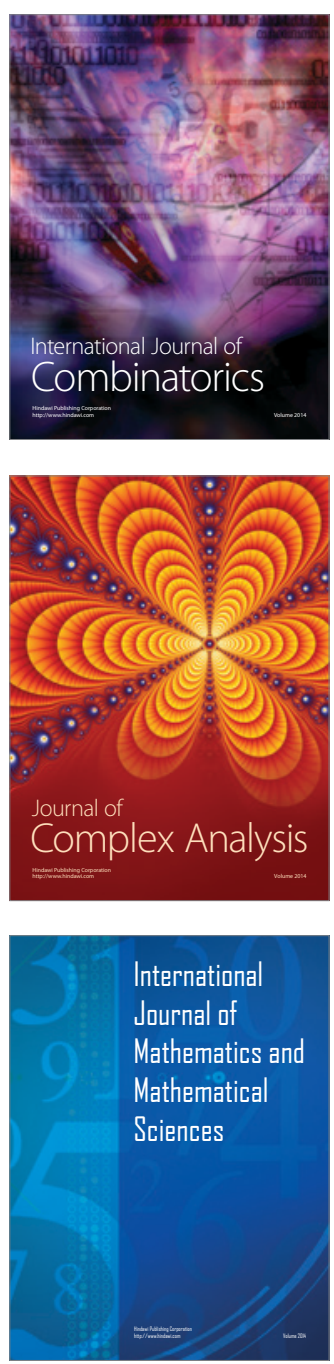
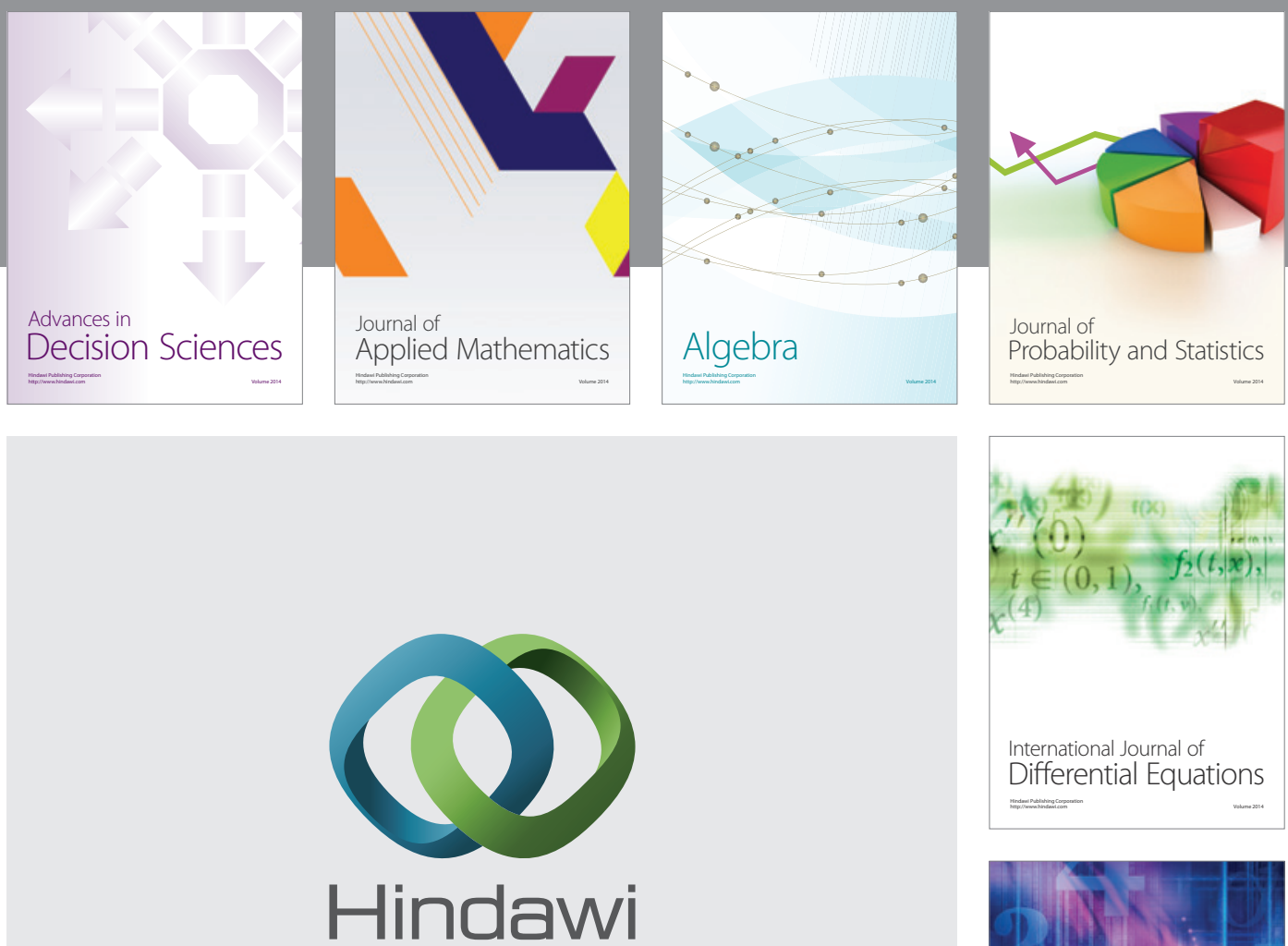

Submit your manuscripts at http://www.hindawi.com
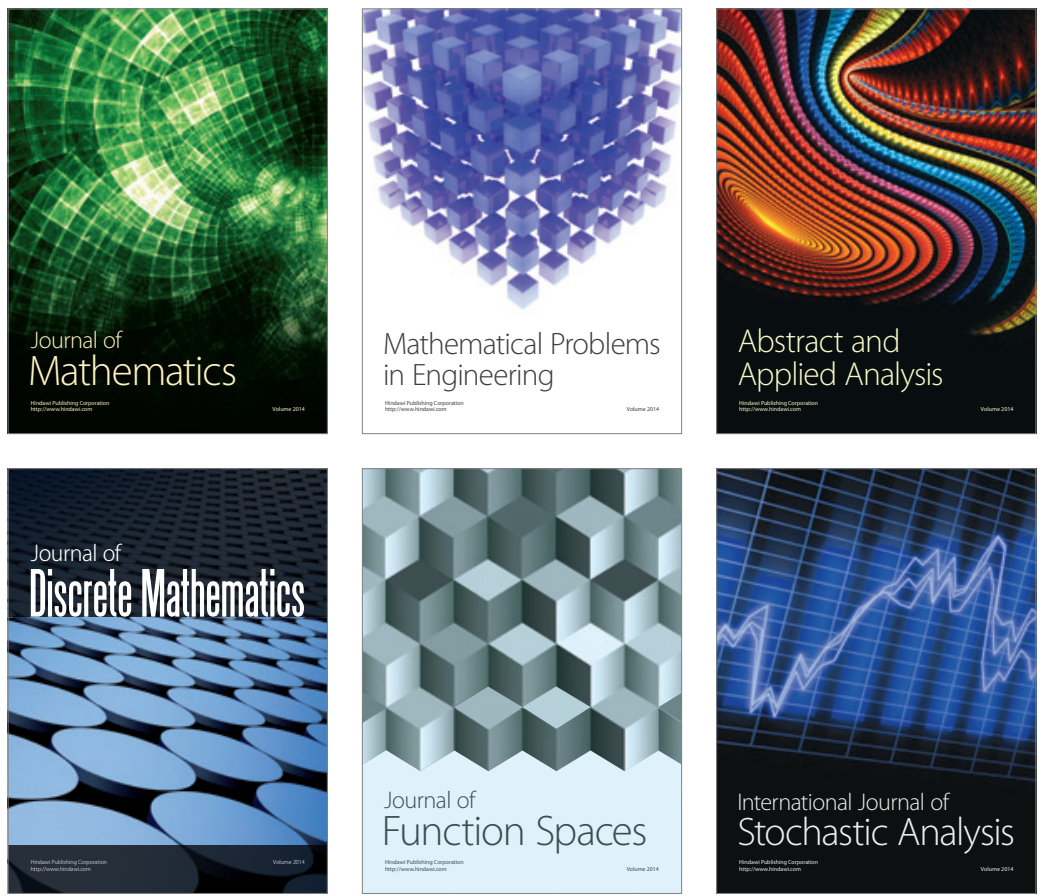

Journal of

Function Spaces

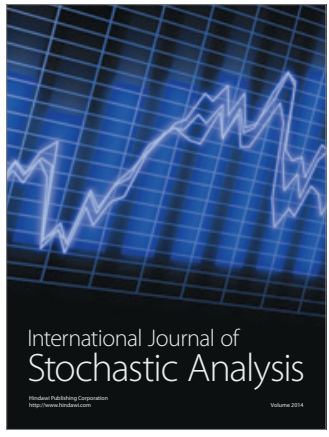

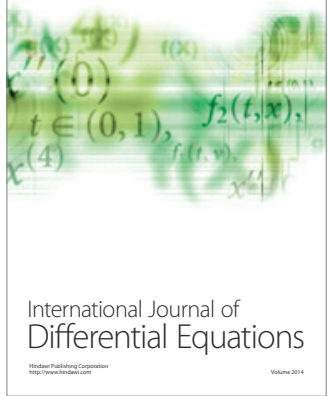
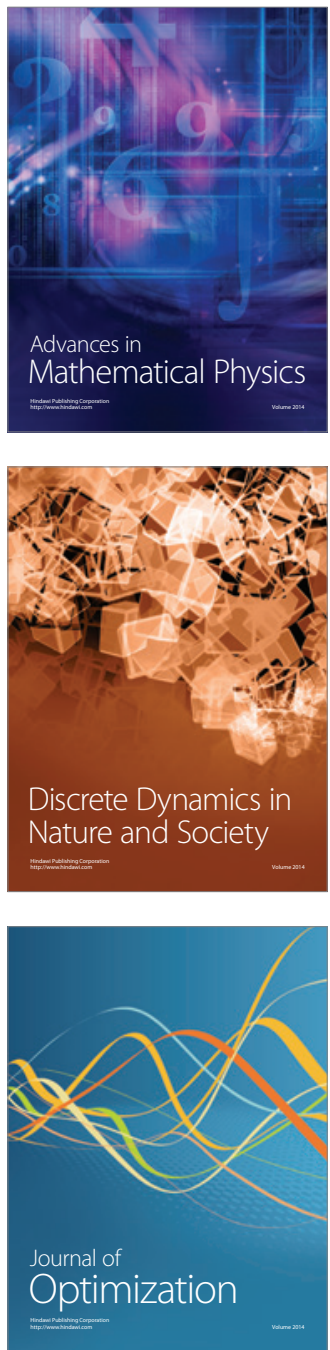